\title{
Elastic Layer on the Elastic Half-Space: The Solution in Matrixes
}

\section{Igor Petrovich Dobrovolsky}

Institute of Physics of the Earth, Russian Academy of Sciences, Moscow, Russia

Email: dipedip@gmail.com

How to cite this paper: Dobrovolsky, I.P. (2021) Elastic Layer on the Elastic Half-Space: The Solution in Matrixes. Open Access Library Journal, 8: e7191.

https://doi.org/10.4236/oalib.1107191

Received: January 27, 2021

Accepted: February 21, 2021

Published: February 24, 2021

Copyright $\odot 2021$ by author(s) and Open Access Library Inc.

This work is licensed under the Creative Commons Attribution International License (CC BY 4.0).

http://creativecommons.org/licenses/by/4.0/

\begin{abstract}
If to apply bidimensional Fourier's transform to homogeneous system of equations of the theory of elasticity, then we will receive system of ordinary differential equations. The general solution of this system contains 6 arbitrary constants and allows to solve problems for the layer and the multilayer environment. It is shown that it is convenient to do statement and the solution of such tasks in the matrix form. The task for the layer on the elastic half-space is solved. Ways of inverse of Fourier's transformation are considered.
\end{abstract}

\section{Subject Areas}

Theory of Elasticity, Matrixes

\section{Keywords}

Bidimensional Fourier's Transformation

\section{Introduction}

Tens of monographs devoted to the solution of various tasks of the classical theory of elasticity are published. Authors apply different methods of modern mathematics. However, modern mathematical programs for computers give the real chance to effectively apply one more method: statement and the solution of tasks in the matrix form after bidimensional Fourier's transform. This work is devoted to it. We will consider the task for the layer on the elastic half-space which has the particular interest for sciences of the Earth.

\section{Bidimensional Fourier's Transformation}

Bidimensional Fourier's transform will be used in the look

$$
\tilde{f}(\xi, \eta)=\int_{-\infty}^{\infty} \int_{-\infty}^{\infty} f(x, y) \mathrm{e}^{(\xi x+\eta y) i} \mathrm{~d} \xi \mathrm{d} \eta=\int_{0}^{\infty} \int_{-\pi}^{\pi} r f(x, y) \mathrm{e}^{i r \rho \sin \tau} \mathrm{d} \tau \mathrm{d} r
$$


where $i=\sqrt{-1}, \quad r=\sqrt{x^{2}+y^{2}}, \rho=\sqrt{\xi^{2}+\eta^{2}}$ and in the second integral $x=\frac{r}{\rho}(\xi \sin \tau+\eta \cos \tau), \quad y=\frac{r}{\rho}(-\xi \cos \tau+\eta \sin \tau)$.

Then inverse transformation receives the form

$$
f(x, y)=\frac{1}{4 \pi^{2}} \int_{-\infty}^{\infty} \int_{-\infty}^{\infty} \tilde{f}(\xi, \eta) \mathrm{e}^{-(\xi x+\eta y) i} \mathrm{~d} \xi \mathrm{d} \eta=\frac{1}{4 \pi^{2}} \int_{0}^{\infty} \int_{-\pi}^{\pi} \rho \tilde{f}(\xi, \eta) \mathrm{e}^{i r \rho \sin \tau} \mathrm{d} \tau \mathrm{d} \rho
$$

wherein the second integral $\xi=-\frac{\rho}{r}(x \sin \tau+y \cos \tau), \quad \eta=\frac{\rho}{r}(x \cos \tau-y \sin \tau)$.

Transformations of derivatives are defined by formulas

$$
F o\left(\frac{\partial^{n} f(x, y)}{\partial x^{n}}\right)=(-i \xi)^{n} \frac{\partial \tilde{f}}{\partial \xi}, \quad F o\left(\frac{\partial^{n} f(x, y)}{\partial y^{n}}\right)=(-i \eta)^{n} \frac{\partial \tilde{f}}{\partial \eta}
$$

The following representations of Bessel functions are useful to inverse transformation of Fourier

$$
\int_{-\pi}^{\pi} \mathrm{e}^{\mathrm{i} z \sin \tau} \mathrm{d} \tau=2 \pi J_{0}(z), \int_{-\pi}^{\pi} i \sin (\tau) \mathrm{e}^{i z \sin \tau} \mathrm{d} \tau=-2 \pi J_{1}(z), \int_{-\pi}^{\pi} \cos (\tau) \mathrm{e}^{i z \sin \tau} \mathrm{d} \tau=0
$$

\section{Displacements and Stresses}

Homogeneous equations of balance of homogeneous isotropic elastic environment in displacements have the form

$$
\operatorname{drad} \operatorname{div} \boldsymbol{u}+(1-2 v) \nabla^{2} \boldsymbol{u}=0
$$

where $\boldsymbol{u}=[u, v, w]$ is displacement vector, $\nabla$ is the nabla-operator, $v$ is Poisson's coefficient.

If to (3.1) to apply bidimensional Fourier's transform on $x, y$, then the general solution of such system receives the form

$$
\begin{aligned}
& \tilde{u}=\left(\chi A_{1}+\xi z G\right) \mathrm{e}^{-\rho z}+\left(\chi B_{1}+\xi z Q\right) \mathrm{e}^{\rho z} \\
& \tilde{v}=\left(\chi A_{2}+\eta z G\right) \mathrm{e}^{-\rho z}+\left(\chi B_{2}+\eta z Q\right) \mathrm{e}^{\rho z} \\
& \tilde{w}=\left(\chi A_{3}-\rho z G i\right) \mathrm{e}^{-\rho z}+\left(\chi B_{3}+\rho z Q i\right) \mathrm{e}^{\rho z}
\end{aligned}
$$

where $A_{i}$ and $B_{i}$ are arbitrary constants, $G=-\frac{\xi A_{1}+\eta A_{2}-\rho A_{3} i}{\rho}$,

$Q=\frac{\xi B_{1}+\eta B_{2}+\rho B_{3} i}{\rho}, \chi=3-4 v$.

For the first time such solution was received in work [1]. In the matrix type of the transform of movements and stresses take the form

$$
\tilde{\boldsymbol{u}}(z)=\frac{\mathrm{e}^{-\rho z}}{\rho} \cdot U \times A+\frac{\mathrm{e}^{\rho z}}{\rho} \cdot V \times B, \tilde{\boldsymbol{s}}(z)=\frac{\mu \mathrm{e}^{-\rho z}}{\rho} \cdot S \times A+\frac{\mu \mathrm{e}^{\rho z}}{\rho} \cdot P \times B
$$

where $\mu$ is the shear modulus, $\times$ is multiplication of matrixes,

$$
\tilde{\boldsymbol{u}}(z)=\left\|\begin{array}{c}
\tilde{u} \\
\tilde{v} \\
\tilde{w}
\end{array}\right\|, \quad \tilde{\boldsymbol{s}}(z)=\left\|\begin{array}{l}
\tilde{\sigma}_{z x} \\
\tilde{\sigma}_{z y} \\
\tilde{\sigma}_{z z}
\end{array}\right\|, \quad A=\left\|\begin{array}{c}
A_{1} \\
A_{2} \\
A_{3}
\end{array}\right\|, \quad B=\left\|\begin{array}{c}
B_{1} \\
B_{2} \\
B_{3}
\end{array}\right\|, \quad \alpha=1-2 v, \beta=1-v
$$




$$
\begin{aligned}
U=\left\|\begin{array}{ccc}
\chi \rho-\xi^{2} z & -\xi \eta z & \xi \rho z i \\
-\xi \eta z & \chi \rho-\eta^{2} z & \eta \rho z i \\
\xi \rho z i & \eta \rho z i & \rho(\chi+\rho z)
\end{array}\right\|, V=\left\|\begin{array}{ccc}
\chi \rho+\xi^{2} z & \xi \eta z & \xi \rho z i \\
\xi \eta z & \chi \rho+\eta^{2} z & \eta \rho z i \\
\xi \rho z i & \eta \rho z i & \rho(\chi-\rho z)
\end{array}\right\| \\
S=\left\|\begin{array}{ccc}
2 \xi^{2} z \rho-\chi \rho^{2}-\xi^{2} & \xi \eta(2 \rho z-1) & -2 \xi \rho(\rho z+\alpha) i \\
\xi \eta(2 \rho z-1) & 2 \eta^{2} z \rho-\chi \rho^{2}-\eta^{2} & -2 \eta \rho(\rho z+\alpha) i \\
-2 \xi \rho(\rho z-\alpha) i & -2 \eta \rho(\rho z-\alpha) i & 2 \rho^{2}(\rho z+2 \beta)
\end{array}\right\| \\
P=\left\|\begin{array}{ccc}
2 \xi^{2} z \rho+\chi \rho^{2}+\xi^{2} & \xi \eta(2 \rho z+1) & 2 \xi \rho(\rho z-\alpha) i \\
\xi \eta(2 \rho z+1) & 2 \eta^{2} z \rho+\chi \rho^{2}+\eta^{2} & 2 \eta \rho(\rho z-\alpha) i \\
2 \xi \rho(\rho z+\alpha) i & 2 \eta \rho(\rho z+\alpha) i & -2 \rho^{2}(\rho z-2 \beta)
\end{array}\right\|
\end{aligned}
$$

\section{Statement and the Solution of the Problem.}

We consider it expedient to remind that the algebra of matrixes differs from algebra of numbers. The main difference consists that matrix multiplication is noncommutativity.

Let's consider the problem about the elastic layer on the surface of the elastic half-space. To simplify calculations, we will consider Poisson's coefficient identical in the layer and the half-space and $v=1 / 4$. At calculations it is necessary to carry out the main simplification: $\xi^{2}+\eta^{2}=\rho^{2}$.

The layer $-h \leq z \leq 0$ is defined by formulas

$\tilde{\boldsymbol{u}}(z)=\frac{\mathrm{e}^{-\rho z}}{\rho} \cdot U \times A+\frac{\mathrm{e}^{\rho z}}{\rho} \cdot V \times B, \quad \tilde{\boldsymbol{s}}(z)=\frac{\mu \mathrm{e}^{-\rho z}}{\rho} \cdot S \times A+\frac{\mu \mathrm{e}^{\rho z}}{\rho} \cdot P \times B, \quad-h \leq z \leq 0$

and the half-space $z \geq 0$

$$
\tilde{\boldsymbol{w}}(x)=\frac{\mathrm{e}^{-\rho z}}{\rho} \cdot U \times C, \quad \tilde{\boldsymbol{g}}(z)=\frac{m \mu \mathrm{e}^{-\rho z}}{\rho} \cdot S \times C
$$

where $\tilde{\boldsymbol{w}}(x)$ is matrix of displacements, $\tilde{\boldsymbol{g}}(z)$ is matrix of stresses, $m \mu$ is the shear modulus in half-space, $m \neq 1$ and $C$ is matrix of arbitrary constants.

On a layer surface at $z=-h$ the following single forces are possible:

$\sigma_{z z}(x, y,-h)=-\delta(x) \delta(y), \quad \sigma_{z x}(x, y,-h)=\sigma_{z y}(x, y,-h)=0$, matrix is $G$;

$\sigma_{z x}(x, y,-h)=-\delta(x) \delta(y), \quad \sigma_{z z}(x, y,-h)=\sigma_{z y}(x, y,-h)=0$, matrix is $G x$,

$\sigma_{z y}(x, y,-h)=-\delta(x) \delta(y), \quad \sigma_{z z}(x, y,-h)=\sigma_{z x}(x, y,-h)=0$, matrix is $G y$.

where $\delta$ is delta-function.

The mentioned matrixes have the form

$$
G=\left\|\begin{array}{c}
0 \\
0 \\
-1
\end{array}\right\|, \quad G x=\left\|\begin{array}{c}
-1 \\
0 \\
0
\end{array}\right\|, \quad G y=\left\|\begin{array}{c}
0 \\
-1 \\
0
\end{array}\right\|
$$

Thus, we have two boundary conditions: on demarcation of at $z=0$ the continuity of movements and stresses is observed and on the surface of the layer at $z=-h$ single force is applied. As a result, system of equations for definition of matrixes of $A, B$ and $C$ receive the look

$$
\tilde{\boldsymbol{u}}(0)=\tilde{\boldsymbol{w}}(0), \quad \tilde{\boldsymbol{s}}(0)=\tilde{\boldsymbol{g}}(0), \quad \tilde{\boldsymbol{s}}(-h)=G
$$


In the right-hand member of the third Equation (4.4) it is possible to substitute matrixes of $G x$ or $G y$ depending on the objective.

The first two equations of system (4.4) in expanded form have the form

$$
A+B=C, \text { So } \times A+P o \times B=m \cdot \text { So } \times C
$$

where $S o$ and $P o$ are matrixes $S_{\text {И }} P$ at $z=0$.

The system (4.4) has the solution

$$
B=(m-1) \cdot N b \times C, \quad A=N a \times C
$$

where $N b=\left(S o^{-1} \times P o-E\right)^{-1}, N a=E-(m-1) \cdot N b$.

Then the equations for the layer receive the form

$$
\begin{aligned}
& \tilde{\boldsymbol{u}}(z)=\frac{\mathrm{e}^{-\rho z}}{\rho} \cdot U \times N a \times C+\frac{\mathrm{e}^{\rho z}(m-1)}{\rho} \cdot V \times N b \times C \\
& \tilde{\boldsymbol{s}}(z)=\frac{\mu \mathrm{e}^{-\rho z}}{\rho} \cdot S \times N a \times C+\frac{\mu \mathrm{e}^{\rho z}(m-1)}{\rho} \cdot P \times N b \times C
\end{aligned}
$$

and the third equation of system (4.4) receives the form

$$
\frac{\mu \mathrm{e}^{\rho h}}{\rho} \cdot S h \times A+\frac{\mu \mathrm{e}^{-\rho h}}{\rho} \cdot P h \times B=G
$$

where $S h$ and $P h$ are matrixes $S_{\text {и }} P$ at $\quad z=-h$.

Solution of (4.8) is

$$
C=\frac{\rho}{\mu} \cdot\left(\mathrm{e}^{\rho h} \cdot S h \times N a+\mathrm{e}^{-\rho h} \cdot(m-1) \cdot P h \times N b\right)^{-1} \times G
$$

or

$$
\begin{aligned}
& C= \frac{\rho}{\mu} \cdot \mathrm{e}^{-\rho h} \cdot C n \\
&=\frac{\rho}{\mu} \cdot \mathrm{e}^{-\rho h} \cdot \| \quad \frac{3 i \xi\left(4 \rho h(m-1) \mathrm{e}^{-2 \rho h}+2 \rho h m-3 m+4 \rho h\right)}{8 \rho^{3} F} \\
& \text { where } \begin{aligned}
F= &
\end{aligned} \\
&+(m-1)^{2} \mathrm{e}^{-4 \rho h}+(m-1)\left(4 \rho^{2} h^{2} m+5 m+4+8 \rho^{2} h^{2}\right) \mathrm{e}^{-2 \rho h} \\
&+(m+2)(2 m+1)
\end{aligned}
$$

Now solutions for a layer and a half-space take a form

$$
\begin{gathered}
\tilde{\boldsymbol{u}}(z)=\frac{\mathrm{e}^{-\rho(z+h)}}{\mu} \cdot U \times N a \times C n+\frac{\mathrm{e}^{-\rho(h-z)}(m-1)}{\mu} \cdot V \times N b \times C n \\
\tilde{\boldsymbol{s}}(z)=\mathrm{e}^{-\rho(z+h)} \cdot S \times N a \times C n+\mathrm{e}^{-\rho(h-z)}(m-1) \cdot P \times N b \times C n \\
\tilde{\boldsymbol{w}}(z)=\frac{\mathrm{e}^{-\rho(z+h)}}{\mu} \cdot U \times C n, \tilde{\boldsymbol{g}}(z)=m \mathrm{e}^{-\rho(z+h)} \cdot S \times C n
\end{gathered}
$$


It is reasonable to carry out calculations on formulas (4.11) at the numerical values $m$. In the final solution it makes sense to make substitution $z \rightarrow z-h$, and then the layer and the half-space will occupy areas $0 \leq z \leq h$ and $z \geq h$.

\section{The Result}

We will not provide all solutions which can be received on formulas (4.11). Let's give only the formula for displacements in the half-space. Calculations were made at $m=5$ and substitution $z \rightarrow z-h$ was made. Displacements have the form

$$
\tilde{\boldsymbol{w}}(z)=\frac{\mathrm{e}^{-\rho z}}{2 \mu}\left\|\begin{array}{c}
\frac{3 i \xi\left(f_{1} \rho z+f_{2}\right)}{2 \rho^{2} f_{0}} \\
\frac{3 i \eta\left(f_{1} \rho z+f_{2}\right)}{2 \rho^{2} f_{0}} \\
\frac{3\left(f_{1} \rho z+f_{3}\right)}{2 \rho f_{0}}
\end{array}\right\|
$$

where $z \geq h, \quad f_{0}=32 \mathrm{e}^{-4 \rho h}+\left(112 \rho^{2} h^{2}+116\right) \mathrm{e}^{-2 \rho h}+77$,

$$
\begin{aligned}
& f_{1}=(8-16 \rho h) \mathrm{e}^{-2 \rho h}+22, \quad f_{2}=\left(8 \rho h+16 \rho^{2} h^{2}\right) \mathrm{e}^{-2 \rho h}-8 \rho h-15, \\
& f_{3}=\left(16 \rho^{2} h^{2}-24 \rho h+16\right) \mathrm{e}^{-2 \rho h}-8 \rho h+29 .
\end{aligned}
$$

Inverse transformations are carried out by means of section 2 formulas. Let's give two examples for arbitrary $H(\rho)$ function

$$
F O^{-1}(H(\rho))=\frac{1}{4 \pi^{2}} \int_{0}^{\infty} \rho H(\rho) \int_{-\pi}^{\pi} \mathrm{e}^{i r \rho \sin (\tau)} \mathrm{d} \tau \mathrm{d} \rho=\frac{1}{2 \pi} \int_{0}^{\infty} \rho H(\rho) J_{0}(r \rho) \mathrm{d} \rho
$$

and

$$
\begin{aligned}
F O^{-1}\left(\frac{i \xi}{\rho} H(\rho)\right) & =\frac{1}{4 \pi^{2}} \int_{0}^{\infty} \rho H(\rho) \int_{-\pi}^{\pi} \frac{i \xi}{\rho} \mathrm{e}^{i r \rho \sin (\tau)} \mathrm{d} \tau \mathrm{d} \rho \\
& =-\frac{1}{4 \pi^{2} r} \int_{0}^{\infty} \rho H(\rho) \int_{-\pi}^{\pi} i(x \sin \tau+y \cos \tau) \mathrm{e}^{i r \rho \sin (\tau)} \mathrm{d} \tau \mathrm{d} \rho \\
& =-\frac{x}{4 \pi^{2} r} \int_{0}^{\infty} \rho H(\rho) \int_{-\pi}^{\pi} i \sin \tau \mathrm{e}^{i r \rho \sin (\tau)} \mathrm{d} \tau \mathrm{d} \rho \\
& =\frac{x}{2 \pi r} \int_{0}^{\infty} \rho H(\rho) J_{1}(r \rho) \mathrm{d} \rho
\end{aligned}
$$

Calculation of the received integrals takes 1 - 2 seconds of machine time and allows to investigate the decision in detail.

\section{Conclusion}

By the offered method, it is possible to solve several other problems for the layer. The solution in all cases turns out rather simple and is uniform. It is sometimes simpler to solve again the known problem, than to look for its solution in literature. 


\section{Conflicts of Interest}

The author declares no conflicts of interest regarding the publication of this paper.

\section{References}

[1] Sneddon, I.N. and Locket, F.J. (1960) On the Steady State Thermoelastic Problem for the Half-Space and for the Thick Plate. Quarterly of Applied Mathematics, 18, 145-153. https://doi.org/10.1090/qam/111318 